\title{
Pancreas transplant in a young patient: an option
}

\author{
Muhammad Zakria
}

Department of Surgery Hepatobiliary, Liver Transplant, Wapda Medical Complex Lahore, Masood Hospital, Lahore, Pakistan

Background: Pancreas transplant is mainly offered to treat the insulin-dependent diabetes mellitus. In some cases, if there is loss of parenchyma due to trauma or chronic pancreatitis, total pancreatectomy is advised to save the life and for better quality of life. Chronic pancreatitis is an inflammatory condition pancreas transplant is important after pancreatectomy. This is required to overcome the exocrine and endocrine deficiency. We have a patient who falls in this category and she requires pancreas transplant. We have to find the solution for the chronic pancreatitis which requires total pancreatectomy followed by the pancreas transplant.

Methods: A 24-year-old female had severe complaints of pain abdomen. Initially she was managed as acute pancreatitis. Due to repeated attacks of pancreatitis, she went into the stage of chronic pancreatitis. Her radiological investigations were showing as if there was loss of parenchyma of pancreas and dilated pancreatic duct. She underwent pancreaticojejunostomy but even after that she could not find the relief. She still has recurrent attacks of pain, indigestion, vomiting, diarrhea, high blood sugar levels and weakness. Pain radiating to back is more challenging. Coeliac axis block is an option for pain but to control other issues by enzymatic preparations and insulin have failed. Probably we have to think about the transplant of pancreas in such patients.

Results: In patients of chronic pancreatitis, if pain persists despite all the maneuvers and quality of life is badly damaged than we have to remove the pancreas followed by the pancreas transplant.

Conclusions: Pancreas transplant is very important in patients of chronic pancreatitis to improve the quality of life. This is not the first option in these patients but if all the other methods for relieving have failed then we accept it as the last choice.

Corresponding author: Muhammad Zakria

E-mail: zakriastar@yahoo.com

(c) The Korean Society for Transplantation

This is an Open Access article distributed under the terms of the Creative Commons Attribution Non-Commercial License (http://creativecommons.org/licenses/by-nc/4.0/) which permits unrestricted non-commercial use, distribution, and reproduction in any medium, provided the original work is properly cited. 